\title{
Vincular la internacionalización con las prioridades de desarrollo de las instituciones de Educación Superior: una urgencia inaplazable*
}

\author{
Vincular a internacionalização com as prioridades de desenvolvimento das \\ instituições de Educação Superior: uma urgência imprescindível
}

\section{Linking internationalization with the development priorities of Higher Education institutions: an essential urgency}

\author{
SYLVIE DIDOU AUPETIT ${ }^{* *}$
}

$\diamond$

RESUMEN

En la pasada década, los gobiernos e instituciones de educación superior (IES) de América Latina y El Caribe reforzaron sus actividades de internacionalización. Sus resultados fueron incrementales pero no bastaron para cumplir con los objetivos de la agenda de internacionalización definida por la Conferencia Regional de Educación Superior (CRES), convocada en 2008 por la UNESCO. No se vincularon orgánicamente con misiones centrales para las universidades latinoamericanas, tales la promoción de la equidad o de la innovación para fines de desarrollo social y productivo. En ese artículo, analizaremos los logros y los desafíos planteados tanto por el cumplimiento de los objetivos de la CRES como por la necesidad de replantear el proceso de internacionalización y diseñar programas endógenos, más eficientes.
\end{abstract}

Palabras clave: Internacionalización. Educación Superior. América Latina.

\section{RESUMO}

$\mathrm{Na}$ década passada, os governos e instituições de educação superior (IES) da América Latina e do Caribe intensificaram suas atividades de internacionalização. Seus resultados foram incrementais porem não bastaram para atender os objetivos da agenda de internacionalização definida pela Conferência Regional de Educação Superior (CRES), convocada, em 2008, pela UNESCO. Não se vincularam organicamente com missões centrais para as universidades latino-americanas, tais como a promoção da equidade ou da inovação para fins de desenvolvimento social e produtivo. Nesse artigo, analisaremos os ganhos e os desafios identificados tanto pelo cumprimento dos objetivos da CRES como pela necessidade de reimplantar o processo de internacionalização e desenhar modelos externos, mas eficientes.

Palavras-chave: Internacionalização. Educação Superior. América Latina.

\begin{abstract}
In the past decade, Latin America and Caribbean governments and Higher Education Institutions (HEI) consolidated their international activities. They bettered their quantitative results but those were not sufficient to fulfill the objectives of an agenda for internationalization defined during the Regional Conference of Higher Education, organized by UNESCO, 2008. Actually, they don't succeed to articulate internationalization with central institutional missions like promotion of equity or innovations for social and productive development. In this paper, we analyze mainly results and issues in relation with the CRES agenda and also the necessity to revise internationalization process and to design endogenous and more efficient programs.
\end{abstract}

Keywords: Internationalization. Higher Education. Latin America.

\footnotetext{
*Apoyado por la Secretaria de Educación Pública - Subsecretaria de Educación Superior - Dirección General de Educación Superior Universitaria, convenio PADES no 2017-09-0006-091.

**Doctora en Ciencias Sociales, por la Escuela de Altos Estudios en Ciencias Sociales, Paris, Francia. Es investigadora de tiempo completo del Centro de Investigación y Estudios Avanzados (CINVESTAV) en México, responsable de la Cátedra UNESCO sobre Aseguramiento de Calidad y provedores emergentes de educación superior en América Latina y coordinadora general de la Red sobre Internacionalización y Movilidades Académicas y Científicas (RIMAC) del programa de Redes temáticas del CONACYT. El articulo se enmarca en un proyecto apoyado por la Secretaria de Educación Pública - Subsecretaria de Educación Superior - Dirección General de Educación Superior Universitaria, convenio PADES no 2017-09-0006-091. PUCRS. E-mail:<didou@cinvestav.mx>.
} 


\section{INTRODUCCIÓN}

En la pasada década, en América Latina y El Caribe (ALC) las instituciones de educación superior (IES) privadas y públicas fomentaron actividades internacionales con más ahínco. Gobiernos, agencias de cooperación, consorcios, redes y asociaciones universitarias firmaron numerosos acuerdos multilaterales y bilaterales, aunque no todos se tradujeron en actividades concretas. Pese a esa expansión, los organismos que lideran la internacionalización y sus modalidades de acción siguen siendo los mismos que hace diez años. Llevan a cabo actividades responsivas a oportunidades y dependen de recursos gubernamentales o externos, de corta y de mediana duración. Promueven fundamentalmente la movilidad saliente, pese a la retórica sobre la necesaria diversificación de las actividades en vista de garantizar una internacionalización en casa o bien comprensiva. Producen una información sobre el proceso de internacionalización desigual según las actividades, escasamente confiable y desactualizada. Hicieron en suma más cosas pero sin preocuparse por aumentar su pertinencia, ni por producir indicadores de seguimiento. En consecuencia, aunque muchas IES en ALC hayan alcanzado ciertos grados de internacionalidad, las dinámicas de internacionalización no impulsaron transformaciones significativas en su qué hacer.

\section{A UNA DÉCADA DE LA CRES: UNA AGENDA DE OBJETIVOS PARCIALMENTE LOGRADA}

Hace casi una década, la UNESCO convocó la Segunda Conferencia Regional sobre Educación Superior en ALC en Cartagena de Indias, Colombia (CRES, 2008). La Declaratoria y el plan de acción definieron como objetivos, en materia de internacionalización:

- fortalecer los esquemas de acreditación regional;

- incrementar la compatibilidad de los programas de estudio e instituciones y avanzar en propuestas de construcción de sistemas unificados de créditos para facilitar su transferencia;

- regular a los proveedores transnacionales y salvaguardar el carácter de bien público de la educación superior;

- disminuir la fuga de cerebros;

- consolidar la dimensión regional de la movilidad estudiantil;

- apoyar la educación virtual;

- articular los sistemas de información;

- incrementar los dispositivos asociativos en ALC. creando redes, favoreciendo los encuentros entre tomadores de decisión y emprendiendo proyectos comunes de investigación o docencia ${ }^{1}$.

ALC amplió entonces los sistemas de becas para la movilidad estudiantil internacional, mediante préstamos, créditos y donaciones. Si bien esos instrumentos no bastaron para superar el carácter elitista y restringido, en términos sociales, de la movilidad estudiantil, elevaron el número de alumnos que realizaban movilidades cortas en el extranjero. Fortalecieron su circulación, en el marco de sub-bloques o a escala bilateral. Flujos ingentes, aunque limitados, de estudiantes se concentran en polos regionales de atractividad - Cuba, Brasil, Argentina y, en menor medida, México.

Las IES, los gobiernos y las asociaciones universitarias han avanzado, asimismo, en el fortalecimiento de la educación virtual, incluyendo los Massive Online Open Courses (MOOC por el acrónimo en inglés). A escala institucional, la Universidad Técnica Particular de Loja, en Ecuador o el Instituto Tecnológico de Estudios Superiores de Monterrey, en México, brindaron servicios de ese tipo. Gobiernos y asociaciones de universidades crearon universidades a distancia o en línea y apoyaron programas transfronterizos virtuales, enfocados a grupos de población en situación de migración internacional. Ofrecieron segmentos o carreras completas compartidas mediante la provisión de materias, impartidas simultáneamente o en diferido a los estudiantes de varios países. Así, la educación virtual contribuyó a mejorar la calidad de la formación, abatir los costos de la movilidad física y proveer de una experiencia intercultural in situ a estudiantes que no estaban en condiciones de moverse (RAMA, 2012).

Consorcios especializados promovieron la adopción y la discusión de la educación virtual: Virtual $\mathrm{Educa}^{2}$, con el apoyo de la Organización de los Estados Americanos, además de brindar cursos de capacitación y organizar congresos y foros internacionales especializados, auspició la instalación del Observatorio de la Educación Virtual en América Latina y el Caribe ${ }^{3}$, con publicaciones en línea sobre Costa Rica, Venezuela, México, Colombia, Perú y Ecuador. Esas señalaron que las problemáticas centrales del sector eran el aseguramiento de la calidad, la regulación y el abatimiento de la deserción. Advirtieron que "muchas instituciones, comenzaron a recorrer el camino de la internacionalización de sus ofertas, tanto a partir del establecimiento de sedes fuera de sus países para apoyar los procesos de enseñanza, como directamente como ofertas transfronterizas totalmente virtuales. En tal sentido, los sistemas de educación superior, están

\footnotetext{
1 CRES 2008, Declaración final, <http://www.oei.es/historico/salactsi/ cres.htm>.

$2<$ http://virtualeduca.org/> (consultado el: 11 sept. 2017).

$3<$ http://virtualeduca.org/observatorio.htm>. (consultado el: 11 sept. 2017).
} 
aumentando la base geográfica de su matrícula, en la nueva competencia por participar en la educación global, fundamentalmente de aquellos países que han habilitado las ofertas en línea" (RAMA, 2016, p. 28).

Un tercer punto en donde se constatan avances en relación a la agenda de la CRES-2008 es el de la asociatividad. Los tomadores de decisión impulsaron una "diplomacia de cumbres", con sus repercusiones positivas visibilización de prioridades políticas) y negativas (operación de iniciativas concretas). Reforzaron o crearon dispositivos iberoamericanos, interamericanos o birregionales. Las redes universitarias tales la Unión de Universidades de América Latina (UDUAL) como algunas universidades se involucraron en proyectos europeoas, tal el proyecto en curso titulado Credential evaluation centres and recognition procedures in Latin American countries - RECOLATIN ${ }^{4}$. Colectivos académicos crearon observatorios y redes, de alcances subregional o internacional, como el Observatorio sobre Movilidades Académicas y Científicas - OBSMAC, apoyado por el Instituto Internacional de la Unesco para la Educación Superior en América Latina y el Caribe - IESALC de la UNESCO, la Red sobre Internacionalización y Movilidades Académicas y Científicas (RIMAC) financiada por el Consejo Nacional de Ciencia y Tecnología - CONACYT en México ${ }^{5}$ y la Red de Estudios sobre Internacionalización de la Educación Superior en América Latina - RIESAL, respaldada por el Mercosur Educativo (Oregioni y Piñero, 2017).

En contraste, la acreditación regional de los sistemas de educación superior no avanzó sustantivamente. Sin embargo, los lineamientos de agencias regionales latino-americanas como la Red Iberoamericana para el Aseguramiento de la Calidad en la Educación Superior - RIACES o la Red de Agencias Nacional de Acreditación (RANA) del Mercosur Educativo facilitaron la implementación de programas de estudios homogeneizados en las profesiones reguladas (HAUG, 2015). Iniciativas recientes parecen además indicar que la cuestión está recobrando actualidad, con iniciativas con las de la UDUAL: la Asociación diseñó en alianza con la Universidad de la Habana, Cuba, la Universidad Nacional de Córdoba, Argentina, y la Universidad Central del Ecuador una Maestría en Gestión de la Calidad y Evaluación de la Educación Superior e impulsó un Consejo de Evaluación y Acreditación Internacional $(\mathrm{CEAI})^{6}$.

\footnotetext{
4 <http://ec.europa.eu/programmes/erasmus-plus/projects/eplus-projectdetails-page/?nodeRef=workspace://SpacesStore/e128ffc0-2222-438cbcb0-4fa1382e1aa4>.

$5<$ http://www.rimac.mx $>$.

$6<$ https://www.udual.org/principal/ceai-consejo-de-evaluacion-yacreditacion-internacional/>.
}

Un número reducido de universidades latino-americanas $^{7}$ buscaron, por su parte, dar fe de su calidad institucional o de la de sus planes y programas, recurriendo a agencias internacionales de rankings o de acreditación especializadas por área del conocimiento o por función para mejorar su posicionamiento internacional. A la par y contradictoriamente, el costo de los servicios y el proceder de esas agencias fue constantemente criticado por sectores de académicos y autoridades que cuestionan su idoneidad para informar la toma de decisión y clasificar las IES (ORDORIKA y LLOYD, 2013).

De manera similar, las grandes iniciativas de generación de información presentadas en la CRES a saber el Mapa de la Educación Superior en América Latina - MESALC, y, luego, el Espacio Latinoamericano y Caribeño de Educación Superior-ENLACES para generar datos sobre la educación superior en ALC (incluida la cooperación internacional), de manera sistemática y comparativa, generaron propuestas más que productos concretos. Si bien las estadísticas nacionales sobre la movilidad entrante o los convenios mejoraron en varios países, en contraste, permanecen inexistentes o incompletas sobre carreras compartidas o académicos internacionales (DIDOU en YUDKEVICH, 2016 sobre México; FARAH y SCHWARTZMANN, 2015 sobre Brasil).

La necesidad de regular a los proveedores transnacionales constituyó un acuerdo entre los participantes de la CRES. Pese a ello, su presencia en la región se ha reforzado y diversificado (Didou, 2015) principalmente en Brasil (KNOBEL, 2016), en Chile (KREMERMAN, 2011) y en México (ÁLVAREZ, 2015). Algunos consorcios especializados en la educación superior (Laureate) o en la educación virtual al igual que universidades privadas, argentinas, brasileñas, chilenas y mexicanas incrementaron sus matricula en Perú, en Ecuador, en Bolivia o en Centroamérica. Grupos empresariales o incluso aseguradoras y fondos de inversiones compraron establecimientos privados a la par que supermercados, seguradoras o farmacias, compitiendo con los consorcios especializados. Independientemente de que el tópico haya perdido relevancia en la investigación y en el debate político, el cómo asegurar la calidad de ese tipo de suministro, para garantizar la defensa de los consumidores, es una cuestión a retomar, como lo muestran escándalos mediáticos puntuales por ejemplo en Chile ${ }^{8}$.

Finalmente, los representantes de ALC externaron su preocupación por las asimetrías en la circulación

\footnotetext{
7 ABET acreditó en su área de competencia una universidad en Chile, cuatro en Colombia, una en Ecuador, 18 en México (la mayoría del sistema ITESM) y 8 en Perú <http://main.abet.org/aps/accreditedprogramsearch. aspx>. Consultado: 11 sept. 2017.

$8<$ http://piensachile.com/2016/08/quienes-los-duenos-laureate-launiversidad-andres-bello-chile/>.
} 
internacional de los recursos humanos altamente calificados. Conforme con ello, en la pasada década, los gobiernos instrumentaron programas de atracción de académicos extranjeros (Ecuador) o de post-doctorantes internacionales (Brasil y México), de regreso de científicos nacionales que trabajan afuera (Argentina, Ecuador Panamá, Uruguay) y de organización de diásporas científicas (Chile, Costa Rica, Panamá, Perú). La combinación de esas acciones contribuyó a proveer algunas universidades principalmente ubicadas fuera de las capitales de los recursos humanos altamente calificados que requerían para consolidar áreas o líneas de investigación pero no bastó para controlar significativamente la fuga de cerebros. La tasa de permanencia de doctores latinoamericanos graduados en Estados Unidos (es decir de la mayoría del contingente de migrantes calificados) no disminuyó, al ser responsiva a factores estructurales exógenos y a un régimen migratorio global sobre los que las IES tienen escaso control, como los políticos, los económicos y los sociales. La emigración de talentos y de personal "con destrezas" aumentó considerablemente (OIM, 2016), generando redes sociales de profesionistas en el exterior cuyo potencial está apenas empezando a ser utilizado en forma estratégica en Argentina (LUCHILO, 2015) o Costa Rica (MUÑOZ, 2015).

En conclusión, ALC cumplió los propósitos de la agenda de la CRES sólo parcialmente. Le faltó optimizar los dispositivos de reconocimiento, convalidación o transferencia de créditos, títulos y grados, no sólo a escala bilateral sino multi-lateral, superar el monolingüismo académico y estandarizar la duración de los programas de licenciatura, maestría y doctorado en términos internacionales para incentivar la movilidad entrante, todavía muy reducida e insuficientemente reportada. Falló en normar las actividades de los consorcios con fines de lucro, de las sucursales universitarias foráneas, de los organismos internacionales de acreditación y de rankings y de las firmas de consulting internacional. No ejerció un seguimiento suficiente de los programas de cooperación e internacionalización, ni produjo indicadores regionales.

\section{GEO-ESTRATEGIA Y DISPOSITIVOS INNOVADORES PARA LA INTERNACIONALIZACIÓN: ALGUNAS PRÁCTICAS INTERESANTES}

Los especialistas señalaron recientemente la necesidad de sustituir programas de internacionalización orientados hacia fuera por unos de naturaleza comprensiva. Ese tránsito depende de la interconexión entre las actividades de internacionalización, los ejes de desarrollo institucional y las demandas externas. Supone reflexionar sobre para qué y cómo internacionalizar las instituciones en función tanto de sus requerimientos propios como de las oportunidades brindadas por los socios de cooperación internacional, dejando de hacer lo mismo con contrapartes cuyas áreas de competencia son diferentes.

Mejorar la eficacia del proceso implicará de hecho diferenciar geo-estratégicamente los programas de internacionalización y revisar el lugar de los países latinoamericanos en la arquitectura de la cooperación internacional. Hasta ahora, esa diferenciación, basada en la detección de intereses no se aprecia con claridad, salvo en lo referido a la cooperación Sur-Sur (por ejemplo, en Brasil en el marco de las relaciones triangulares de Portugal con sus antiguas colonias en África y América Latina, FRANCA y PADILLA, 2016) y a binomios de cooperación (México-Alemania, CRUZ, 2017; América Latina-Asia, RAMÍREZ, 2017).

Implica asimismo articular los tres procesos de internacionalización que coexisten en paralelo en las IES, a saber el oficial (convenios), el vinculado con una internacionalización académica (redes, proyectos de investigación, enseñanza de idiomas y actividades de internacionalización en casa) y el administrado por las oficinas de asuntos internacionales (relacionado con la movilidad estudiantil). Aunque los directivos, los gestores, los académicos y los estudiantes demanden oportunidades de internacionalización, sus intereses difieren. Si bien esas divergencias no afectan la capacidad de cada sector para alcanzar sus objetivos, sí, dificulta ampliar el radio de proyección y legitimidad de las iniciativas.

Pese a ello, algunos programas innovadores emergieron recientemente, distintos a los centros disciplinarios de excelencia científica que tradicionalmente fueron financiados por agencias de cooperación académica internacional o regional (sobre FLACSO Argentina, ALGAÑARAZ, 2016; sobre la escuela nacional de Física teórica o el Observatorio Sismológico del Sur Occidente de Colombia, el Instituto de Biología Andina de Perú, HODARA, 2014). Esos nuevos dispositivos recibieron fondos de empresas transnacionales y extranjeras (la Universidad Aeronáutica de Querétaro en México recibe por ejemplo apoyos de BOMBARDIER y de BOINGHERNÁNDEZ, 2011). Vincularon la internacionalización con procesos de emprededurismo con empresas extranjeras o respaldaron proyectos de promoción de la equidad, con énfasis en la inclusión de los pueblos indígenas (mapuches en Chile, WILLIAMSON y NAVARRETE, 2014).

Lo anterior corrobora que, para que la internacionalización se vuelva una práctica legítima y sostenible a nivel institucional, debe contribuir a la renovación de los perfiles de competencias profesionales, al diseño de proyectos de vinculación productiva y social y a la incorporación de componentes prácticos en las formaciones. Debe colaborar con el proceso, lento 
y discontinuo, de integración de un espacio regional latino- o iberoamericana de educación superior. A la par, debe diversificar sus contrapartes, tanto en la dimensión Norte-Sur como en las Sur-Sur y triangular, en perspectivas solidarias $\mathrm{y} / \mathrm{o}$ comerciales. Asia del Pacifico, principalmente Japón, Corea y China, ofrecen oportunidades insuficientemente exploradas, sobre todo para los países latinoamericanos que tienen relaciones con ellas, por cuestiones migratorias (Japón con Perú y con Brasil) o por su inserción en organismos de la Cuenca del Pacifico (Chile, México) - Ramírez Bonilla, 2016. África como espacio de oportunidades para la colaboración universitaria, hasta ahora, ha sido aprovechado esencialmente por países latinoamericanos con una población afrodescendiente: Cuba, Brasil, Colombia y Venezuela empujan proyectos de cooperación técnica, de apoyos solidarios y de diplomacia cultural focalizada en la enseñanza del español (BIEL, 2016).

Para acelerar esos cambios de perspectiva, seria relevante documentar mejor prácticas de interés en cuanto a redes y a dispositivos endógenos de cooperación, según los tipos de establecimientos de educación superior. Sería necesario analizar su articulación orgánica con sus perfiles, según sean orientados a la docencia, la investigación o la vinculación y monitorear el funcionamiento de instituciones de educación superior, creadas en la pasada década o en la actual, con proyección regional o internacional: la Universidad Federal de la Integración Latino americana (UNILA), instalada en Foz de Iguazu, Brasil tiene así un radio de influencia, esencial - aunque no exclusivamente, en los países que convergen en torno a la triple frontera de Paraguay, Argentina y Brasil y en Mercosur. Ofrece enseñanza bilingüe portuguésespañol con títulos validados en el espacio del Mercosur (BRACKMAN, 2010). La Universidad de Integración Internacional de Lusofonía Afro-brasileña (UNILAB) en el estado de Ceará, Brasil, recibe números importantes de estudiantes de las excolonias portuguesas en la África lusófona y tiene un componente internacional, desde sus estudios de factibilidad (LIMA, 2017).

\section{MÁS ALLÁ dE LA AGENDA PROGRAMADA: INSTITUCIONALIZACIÓN Y GOBERNANZA DE LA INTERNACIONALIZACIÓN}

Las economías latinoamericanas pasaron por ciclos de bonanza en la segunda década del 2000. Los gobiernos de Brasil, México, Ecuador incrementaron significativamente sus recursos para la internacionalización. No obstante, el proceso tuvo alcances reducidos en las IES y no dejó de representar un asunto polémico en la arena universitaria regional por cuestiones laborales, sindicales y políticas. Los profesores de tiempo "repleto" y muchos académicos la resintieron como una presión adicional a las que ya están sometidos, en un escenario dominado por la evaluación como "organizador" de la profesión académica.

En paralelo, grupos, asociaciones, redes estudiantiles o académicas y sindicatos, principalmente en Brasil, Argentina y México, denuncian el carácter asimétrico y dependiente del fenómeno en relación a los países dominantes y, por ende, su cariz "endocolonial" (FRANCOIS et al., 2016). En esa perspectiva, si bien la internacionalización representa un elemento central en la retórica de las autoridades sectoriales e institucionales, no está institucionalizada ni en tanto objeto legítimo de políticas públicas e institucionales.

Ante el consenso endeble que genera la internacionalización, en términos políticos, habría que buscar fortalecer, en ver de una cooperación académica supeditada, inscrita en un marco de cooperación SurNorte, una recíproca. Casi una década después de la CRES 2008, el espacio latino-americano de educación superior se ha consolidado, principalmente en sub-bloques como MERCOSUR (Programa MARCA), mediante movilidades bi o multilaterales y transfronterizas, la firma de convenios y la celebración de eventos de concertación política sobre prioridades regionales en educación superior. Pero le falta tener una mayor proyección, principalmente hacia el Caribe, Asia y África.

En términos institucionales, el proceso de internacionalización universitaria debe incrementar su legitimidad. Para ello, es indispensable:

- Diseñar acciones de formación e información que incentiven mayores compromisos de los distintos actores involucrados en la operación o en la ejecución de las actividades de internacionalización.

- Contar con documentar estratégicos pero concertados para definir no solo prioridades sino condiciones internas de apoyo.

- Propiciar una adecuada gestión de las actividades internacionales para facilitar la producción de conocimientos en redes internacionales, los intercambios con fines de docencia, de investigación, de vinculación y de aprendizaje, la constitución de redes y la internacionalización del curriculum.

Para optimizar la administración de los programas de internacionalización, se requeriría además atender los disfuncionamientos en los procesos de gobernanza institucional de la educación superior que, en los últimos años, han sido profusamente identificados por los especialistas (ACOSTA, CAMOU y ATAIRO, 2015 sobre México y Argentina; BRUNNER y GANGA, 2016 sobre Chile). Los que más afectan la internacionalización consisten en: 
- Las rupturas en las cadenas de mando entre las autoridades, los cuadros medios y la burocracia a cargo de supervisar los trámites en materia de asignación de los recursos conseguidos mediante organismos externos, incluyendo los internacionales, de reconocimiento de créditos, de firma y operación de los convenios, de contratación, temporal o definitiva, de profesores internacionales .

- Las contradicciones entre las decisiones de política y las regulaciones de la educación superior y las IES, principalmente las que conciernen las condiciones de ejercicio profesional y, en particular, la exclusividad de los docentes e investigadores.

- Los escasos apoyos financieros institucionales a la internacionalización, en una situación en la que una proporción elevada de los presupuestos está destinada al pago de nóminas y en la que la viabilidad de las iniciativas depende de apoyos externos suministrados por los agencias o internacionales.

Se requeriría igualmente formalizar la posición jerárquica en los organigramas institucionales y las atribuciones de las oficinas encargadas de los asuntos internacionales, en las universidades públicas y privadas. Aunque los estudios sobre la administración de la internacionalización son pocos o desactualizados, relevaron una tendencia a empoderar esos dispositivos, pese a diferencias en sus denominaciones, en sus medios y en sus competencias. Las oficinas de asuntos internacionales, por lo menos en las IES que tradicionalmente los fomentan, juegan un rol de articulador y prestan servicios de apoyo a estudiantes, académicos y autoridades. Pero, aunque gocen de una buena aceptación por parte de sus "usuarios", salvo excepciones, carecen de presencia en términos políticos, independientemente de que su visibilidad mediática se haya incrementado (DIDOU, 2017).

Desgraciadamente, en los otros segmentos de los sistemas nacionales de educación superior, escasean los análisis sobre la internacionalización de los establecimientos tecnológicos, politécnicos e interculturales.

En una circunstancia en la que las políticas de austeridad están golpeando ya las IES, es alto el riesgo de crisis múltiples (internas y externas) en la educación superior. Ajustes preventivos para reducir la incidencia de conflictos en torno a la internacionalización concernirían la racionalización y la alineación de las actividades sobre las prioridades de desarrollo institucional, local y nacional. Para ello, se requeriría dejar atrás el tono triunfalista que caracteriza muchas declaraciones sobre la internacionalización: esas hiper-valoran resultados por cierto incrementales, pero poco significantes en términos de cambio institucional y de posicionamiento regional. Aunque los números de estudiantes entrantes aumentaron en ALC, su alza no debe disimular que la región sigue siendo de baja atractividad, incluso para los latinoamericanos y con una movilidad hacia fuera limitada, y, por lo tanto, elitista. La movilidad saliente por su parte está soportada por unos pocos países: Brasil, México y Colombia, en conjunto, envían más del $40 \%$ del total de latino-americanos en intercambio internacional. En consecuencia, la matrícula internacional no alcanzaba el $1 \%$ de la matricula regional, una tasa realmente baja a escala internacional (UNESCO, 2014).

\section{AMÉRICA LATINA ANTE LA ADMINISTRACIÓN DE DONALD TRUMP: LOS DESAFÍOS DE LA MIGRACIÓN DE RETORNO}

Actualmente, el desafío internacional de mayor preocupación para ALC tiene que ver con las decisiones de política internacional del presidente Donald Trump en Estados Unidos. En enero 2016, sus declaraciones antimigratorias suscitaron incertidumbre sobre el futuro de los programas de movilidad estudiantil o académica y sobre las oportunidades de trabajo ofrecidas a doctores latinoamericanos. Pusieron fin a un ciclo de reforzamiento de la cooperación académica entre América Latina y Estados Unidos. En la segunda década del siglo XXI, en efecto, Estados Unidos había acrecentado su dominancia en tanto principal polo de recepción de movilidad: pasó de recibir a 65632 estudiantes latinoamericanos en 2009-2010 a 86378 en 2014-2015 (31.6\% de aumento en el periodo) - IIE, 2016, alcanzando una tasa de participación en el total de movilidad entrante a Estados Unidos del $8.9 \%$ en 2014-20159. En 2014, el promedio mundial de movilidad internacional a Estados Unidos era del 19\% del total, pero el regional del $33 \%{ }^{10}$, siendo ese país el principal destino de los estudiantes salientes en 25 de 42 naciones de ALC.

En esa situación, el plan migratorio de Estados Unidos y la revocación del Programa de Acción Diferida para los Llegados en la Infancia (DACA, por sus siglas en inglés), lanzado en 2012 por el gobierno del expresidente Barack Obama ${ }^{11}$, suscitó inquietudes y repudio en América Latina. En México, el país más afectado por el tamaño de sus flujos estudiantiles y académicos hacia y desde Estados Unidos, varias instituciones de educación

\footnotetext{
$9<$ http://www.iie.org/Research-and-Publications/Open-Doors/Data/ International-Students/All-Places-of-Origin/2013-15>.

$10<\mathrm{http}$ ///www.uis.unesco.org/Education/Pages/international-student-flowviz.aspx $>$.

$11<$ http://conacytprensa.mx/index.php/ciencia/humanidades/18432dreamers-ante-revocacion-daca $>$.
} 
superior, públicas y privadas anunciaron medidas para incorporar a estudiantes amenazados de deportación o de no renovación de sus visas. La ANUIES diseñó el Programa Universitario Emergente Nacional para la Terminación de Estudios Superiores (PUENTES), con carácter extraordinario y temporal. Su objetivo es facilitar el ingreso de jóvenes estudiantes que vivían y estudiaban en EUA pero que, por su condición migratoria, fueron obligados a concluir sus estudios en instituciones de educación superior mexicanas. Cuenta con la participación de más de 300 IES, públicas y privadas ${ }^{12}$. El Senado de la República, el 28 de febrero 2017, aprobó el dictamen que reforma la Ley General de Educación, con disposiciones para simplificar la revalidación de estudios realizados en el extranjero ${ }^{13}$.

Independientemente de su tamaño, el previsible incremento de una migración estudiantil inversa llevará a las IES latino-americanas a revisar sus calendarios de admisión, a diferenciar sus procesos de enseñanza y a ampliar sus infraestructuras y plantillas docentes. Las obligará a interculturalizar su atención a poblaciones jóvenes, en condición de trauma y con un sentido de pertenencia al país de origen no siempre firme, dependiendo de la edad y de las condiciones en las que migraron. Implicará que los gobiernos reordenen sus programas de becas al extranjero y consideren alternativas a su "dependencia académica" de Estados Unidos, naturalizada al punto de haber sido escasamente cuestionada a diferencia de la dependencia intelectual. Para hacerlo en un plazo razonable, los países de la región tendrán que formar mejor a los gestores, reinsertar a los académicos en los procesos decisionales relativos a internacionalización, definir orientaciones generales sobre con quienes cooperar, en qué ámbitos y en qué áreas disciplinarias y diseñar programas pilotos a escala institucional.

\section{DE LOS BUENOS PROPÓSITOS A LAS RESPUESTAS EFECTIVAS}

La internacionalización es sin duda un tema en discusión, conceptual (TANGELSON, 2014) y operativamente. En esa última dimensión y en aras de una reflexión sobre las políticas de gobernanza universitaria, conviene preguntarse si las IES en ALC son organizaciones susceptibles de reinventarse y en qué condiciones de inducción y autonomía pueden conseguirlo. En relación a la internacionalización, llevaría a recomendar que integren balances críticos y elaboren propuestas concretas acordes

\footnotetext{
$12<$ http://puentes.anuies.mx/public/site/ies-participantes/>.

$13<$ http://comunicacion.senado.gob.mx/index.php/informacion/ boletines/87-internacional/34623-senado-aprueba-reforma-a-ley-deeducacion-que-facilita-revalidacion-de-estudios-a-migrantes.html>.
}

con una visión a futuro, en ejercicios auto evaluativos similares a los hechos en Colombia por la Asociación Colombiana de Universidades - ASCUN (2014).

Lo anterior sólo es factible pasando de una internacionalización aspiracional a una pragmática y de carácter endógeno es decir arraigado en los territorios institucionales y legitimo para todos los actores de la vida universitaria. Una IES de proximidad, enfocada a la enseñanza en licenciatura, tiene oportunidades de internacionalización distintas a las que tienen los institutos de posgrado y los centros de investigación. Las experiencias de internacionalización que persigan fines de optimización organizacional, de promoción de la equidad social y de innovación productiva deben por lo tanto ser multiplicadas, en torno a líneas prioritarias que otorguen a la internacionalización un carácter de "factibilidad transformativa". Lo anterior implica amarrar mejor la internacionalización en los establecimientos, creando entornos amigables de inducción para incluir a los "excluidos" del proceso mediante dispositivos motivacionales.

Sería recomendable, en ese mismo tenor, que las IES detecten los obstáculos organizacionales que limitan su participación, identifiquen autocríticamente sus fortalezas para capitalizarlas mejor y aprendan de otros, compartiendo experiencias. Lo sería que articulen sus programas de internacionalización con asuntos nodales para la educación superior en toda ALC. Entre esos, destacan la internacionalización del curriculum para la formación de una nueva ciudadanía y la adquisición de competencias interculturales (COSTA et al., 2016), la redistribución de oportunidades de movilidad a las poblaciones en situación de vulnerabilidad educativa en la educación superior y la consolidación de procesos democráticos y de naturaleza claramente académica de internacionalización. Sólo si se aboca a la resolución de esas problemáticas, la internacionalización será un catalizador de cambio, no un maquillaje conceptual (BENEITONE, 2014).

\section{REFERENCIAS}

ACOSTA, Adrián; CAMOU, Antonio; ATAIRO, Daniela. Gobernabilidad y democracia en la universidad pública latinoamericana: Argentina y México en perspectiva comparada. Argentina, CLACSO, 2015. Disponible en: <http:// biblioteca.clacso.edu.ar/clacso/becas/20150303042220/ PREMIO_KAAC_VersionrevisadaFINAL_27_02_2015.pdf $>$. Acceso al: 24 oct. 2017.

ALGAÑARAZ, Victor. Between scientific autonomy and academic dependency: private research institutes under dictatorship in Argentina (1976-1983). The case of FLACSO. En: BEIGEL, Fernanda. The politics of academic autonomy in Latin America. New York: Routledge, 2016. Disponible en: 
<https://books.google.com.mx/books?hl=es\&lr=\&id=LOSqC wAAQBAJ\&oi=fnd\&pg=PA249\&dq=Beigel+FLACSO\&ots= GwtFaSQ1_J\&sig=2N4_E_pDvwpmK8UUM7aAGXe_34Y\# $\mathrm{v}=$ onepage $\& \mathrm{q}=$ Beige $1 \% \overline{2} 0$ FLACSO\&f=false $>$. Acceso al: 24 oct. 2017.

ÁLVAREZ, Germán. La educación superior transnacional en México. Página web RIMAC (Red sobre Internacionalización y Movilidades Académicas y Científicas), 2015. Disponible en: $<$ http:/www.rimac.mx/la-educacion-superior-transnacional-enmexico/>. Acceso el: 24 oct. 2017.

ASCUN. Problemas nodales e la internacionalización de la educación superior en Colombia, principios orientadores y lineamientos para la construcción de política pública. Colombia: Asociación Colombiana de Universidades. 2014. Disponible en: <http://secretariageneral.univalle.edu.co/ consejo-academico/temasdediscusion/2014/Documentos de interes general/Lecturas Internacionalizacion/Documento final_mesa_internacionalizacion_agosto_2013.pdf $>$. Acceso el: $2 \overline{4}$ oct. $\overline{2017}$.

BENEITONE, Pablo. De la cooperación internacional universitaria a la internacionalización de la educación superior: ¿cambio de paradigma o maquillaje conceptual? En: TANGELSON, Guillermo (Comp.). Desde el sur: miradas sobre la internacionalización. Buenos Aires: Ediciones de la UNLA - Universidad Nacional de Lanús, 2014. Disponible en: <http:/www.cinda.cl/wp-content/uploads/2014/09/Desdeel-sur-miradas-sobre-la-internacionalizaci\%C3\%B3n.pdf $>$. Acceso el: 24 oct. 2017.

BIEL, Alejandre. Reflexión sobre la situación de enseñanza del español en Africa subsahariana y los factores que impulsan su expansión. Ogigia. Valladolid, n. 20, p. 49-69. 2016. Disponible en: <http://www.ogigia.es/index.php/ogigia/article/view/77>. Acceso el: 24 oct. 2017.

BRACKMANN, Marta. Internacionalização da educação superior e política externa brasileira: estudo da criação da Universidade Federal da Integração Latino-Americana (UNILA). Tesis (de maestría) - Pontificia Universidade Católica do Rio Grande do Sul, Porto Alegre, 2010. Disponible en: <http://meriva.pucrs.br:8080/dspace/handle/10923/1994>. Acceso el: 24 oct. 2017.

BRUNNER, José; GANGA, Francisco. Dinámicas de transformación en la educación superior latinoamericana: Desafíos para la gobernanza. Opción, año 32, n. 80, p. 12-35, 2016. Disponible en: <http://200.74.222.178/index.php/opcion/ article/view/21404/21225>. Acceso el: 24 oct. 2017.

COSTA, Marilia; USTÁRROZ, Elisa. Impactos da internacionalização da educação superior na docencia universitária: construindo a cidadania global por meio do currículo globalizado e das competências interculturais. Em Aberto, Brasília, v. 29, n. 97, p. 35-46, 2016. Disponible en: <http://emaberto.inep.gov. br/index.php/emaberto/article/view/2949>. Acceso el: 24 oct. 2017.

CRUZ, Laura. La internacionalización de la educación superior mexicana, una mirada a las cooperaciones universitarias entre Alemania y México en el área de Educación. Disponible en: <http://www.mufm.fr/sites/mufm. univ-toulouse.fr/files/evenement/symposium/ponencias/laura patricia_cruz_ruiz.pdf $>$. Acceso el: 24 oct. 2017.
DIDOU, Sylvie. La internacionalización de la educación superior en América Latina: Transitar de lo exógeno a lo endógeno: resumen ejecutivo. México: UDUAL, 2017. Disponible en: <https://www.udual.org/pdf/Internacionalizacion $\%$ 20exogeno\%20endogeno.pdf>. Acceso el: 24 oct. 2017.

DIDOU, Sylvie. Attraction, Integration, and Productivity of international Academics in Mexico. En: YUDKEVICH, M.; ALTBACH, P.; RUMBLEY, L. (Ed.). International Faculty in Higher Education. Comparative Perspectives on Recruitment, Integration and Impact. E.U.: Routledge. 2016.

DIDOU, Sylvie. Fourniture transnationale de services d'enseignement supérieur en Amérique latine. Une première approche de ce phénomène. Revue Tiers Monde, Francia, v. 223, n. 3, p. 111-126, 2015. Disponible en: <http://www.cairn. info/resume.php?ID_ARTICLE=RTM 223 0111>. Acceso el: 24 oct. 2017.

FRANCOIS, Emmanuel; AVOSEH, Mejai; GRISWOLD, Wendy (Ed.). Perspectives in transnational higher education. Rotterdam: Sense Publishers, 2016. Disponible en: <https:// www.ohio.edu/tels/upload/About-transnational-education. pdf>. Acceso el: 24 oct. 2017.

FARAH, Luisa; SCHWARTZMAN, Simon. Migrations des personnes hautement qualifiées au Brésil. De l'isolement à l'insertion internationale? Revue Brésil(s), Paris, 7, p. 147171, 2015. Disponible en: <https://bresils.revues.org/1516\# quotation>.

FRANCA, Thais; PADILLA, Beatriz. Acordos bilateral de cooperação acadêmica entre Brasil e Portugal: Internacionalizaçao ou (pós)colonização universitária? Universidades, año 5, n. 16/69, p. 57-73, 2016. Disponible en: <http:// publicaciones.udual.org/doss69-4.html>. Acceso el: 24 oct. 2017.

HAUG, Guy. Aspectos de la Internacionalización de las Agencias de Calidad Universitaria. Foro+U, Universidad Andrés Bello, Chile, 2015. <http://observatoriouniversitariodechile.org/chile/ index.php/opinion-publica/guy-haug/>.

HERNÁNDEZ, Juana. Transferencia de conocimiento en la industria aeroespacial mexicana: el caso de bombardier aeroespacial, Querétaro, Revista de Economía del Caribe, Colombia, n. 7, p. 231-269. 2011. Disponible en: <http:// rcientificas.uninorte.edu.co/index.php/economia/article/ view/2044>. Acceso el: 24 oct. 2017.

HODARA, Joseph. Ciencia en la periferia de la periferia: hacia la formación de colegios virtuales. Estudios Interdisciplinarios de América Latina y el Caribe, Tel Aviv, v. 14, n. 1, 2014. Disponible en: <http://www7.tau.ac.il/ojs/index. php/eial/article/view/933/969>. Acceso el: 24 oct. 2017.

IIE. Open Doors. Data and Fact Sheet. 2016. Disponible en: $<$ http://www.iie.org/Research-and-Publications/Open-Doors/ Data/International-Students/Leading-Places-of-Origin\#. WBEuo2AzVx>. Acceso el: 24 oct. 2017.

KREMERMAN, Marco. Dos holdings de educación superior concentran más alumnus que 16 universidades estatales. El Mostrador, Santiago, 25 jul. 2011. Disponible en: <http:// www.elmostrador.cl/noticias/opinion/2011/07/25/dos-holdingsde-educacion-superior-concentran-mas-alumnos-que-16universidades-estatales/>. Acceso el: 24 oct. 2017. 
KNOBEL, Marcelo. In Brazil the For-Profit Giants Keep Growing. Inside Higher ED, Washington, 18 jul. 2016. Disponible en: <https://www.insidehighered.com/blogs/world-view/ brazil-profit-giants-keep-growing>. Acceso el: 24 oct. 2017.

LIMA, Francisca. Voces y contra-voces de un discurso universitario de habla portuguesa: la cooperación internacional en la Universidad de Integración Internacional africanos de habla portuguesa-brasileña - UNILAB. São Paulo: Universidade Nove de Julho, 2017. Disponible en: $<$ http://bibliotecatede. uninove.br/handle/tede/1668>. Acceso el: 24 oct. 2017.

LUCHILO, Lucas. Más allá de la fuga de cerebros: movilidad, migración y diásporas de argentinos calificados. Buenos Aires: Eudeba. 2015.

MUÑOZ, Luis. Vinculación institucional en red con la emigración altamente calificada. Percepciones de costarricenses en el extranjero. Cuadernos de inter-c-a-mbio sobre Centroamérica y el Caribe, v. 12, n. 1, p. 27-51,2015. Disponible en: <http://www.redalyc.org/pdf/4769/476947243003.pdf>. Acceso el: 24 oct. 2017.

OIM. Migración calificada y desarrollo: Desafíos para América del Sur. Buenos Aires: OIM, 2016. Disponible en:<http://www. iom.int/sites/default/files/press_release/file/OIM-MigracionCalificada-en-America-del-Sur.pdf $>$. Acceso el: 24 oct. 2017.

ORDORIKA, Imanol; Lloyd, Marion. A decade of international university rankings: a critical perspective from Latin America. In MAROPE, Priscilla; Wells, Peter; Hazelkorn, Ellen (Eds.), Rankings and accountability in Higher Education: uses and misuses. Paris: UNESCO Publishing. 2013. Disponible en: $<$ http://unesdoc.unesco.org/images/0022/002207/220789e. pdf>. Acceso el: 24 oct. 2017.

OREGIONI, María; PIÑERO, Fernando. Las redes como estrategia de internacionalización universitaria en el Mercosur. El caso de la RIESAL (2013-2017). Integración y Conocimiento, Córdoba, v. 1, n. 6, p. 114-133. 2017. Disponible en: <https:// revistas.unc.edu.ar/index.php/integracionyconocimiento/issue/ view/1439/showToc>. Acceso el: 24 oct. 2017.
RAMA, Claudio. La fase actual de expansión de la educación en línea o virtual en América Latina. Universidades, México, n. 70, p. 27-39, 2016. Disponible en: <http://www.redalyc.org/ pdf/373/37348529004.pdf>. Acceso el: 24 oct. 2017.

RAMA, Claudio. La internacionalización de la educación a distancia en América Latina. Revista Cuestiones de Sociología, Argentina, n. 8, p. 63-76, 2012a. Disponible en: $<$ www.cuestionessociologia.fahce.unlp.edu.ar/article/view/ CSn08a04>. Acceso el: 24 oct. 2017.

RAMÍREZ, Juan (Coord.). La internacionalización en las instituciones de educación superior mexicanas. Experiencias de vinculación con Asia. México: RIMAC/CINVESTAV, 2017.

RAMÍREZ, Juan. Iniciativas institucionales latinoamericanas para generar conocimiento sobre Asia. Universidades, México, n. 69, p. 9-21, 2016. Disponible en: <http://publica ciones.udual.org/pdf/Universidades69.pdf>. Acceso el: 24 oct. 2017.

TANGELSON, Guillermo (Comp.). Desde el sur: miradas sobre la internacionalización. Buenos Aires: Ediciones de la UNILA - Universidad Nacional de Lanús, 2014.

UNESCO. Global flow of tertiary-level students. 2014. Disponible en: <http://uis.unesco.org/en/uis-student-flow>. Acceso el: 24 oct. 2017.

WILLIAMSON, Guillermo; Navarrete, Susana. Cooperación internacional y educación intercultural bilingüe en Chile: cuatro casos en la educación superior. Revista Mexicana de Investigación Educativa, México, v. 19, n. 60, p. 19-43, 2014. Disponible en: <http://www.scielo.org.mx/scielo.php?pid=S14 $0566662014000100003 \&$ script $=$ sci_arttext\&tlng=pt $>$. Acceso el: 24 oct. 2017.

Recebido em 30-10-2017. Aprovado em 27-12-2017. 